\title{
Self-assembly low dimensional inorganic/organic heterojunction nanomaterials
}

\author{
QIAN XueMin $^{1,2}$, LIU HuiBiao $^{1} \&$ LI YuLiang ${ }^{*}$ \\ ${ }^{1}$ CAS Key Laboratory of Organic Solids, Beijing National Laboratory for Molecular Sciences (BNLMS), Institute of Chemistry, Chinese Academy \\ of Sciences, Beijing 100190, China; \\ ${ }^{2}$ Department of Physics, Faculty of Science, Harbin Institute of Technology, Harbin 150001, China
}

Accepted February 2, 2013; accepted February 21, 2013; published online June 24, 2013

\begin{abstract}
One dimensional inorganic/organic heterojunction nanomaterials have gained extensive attention in materials science because of their outstanding optical and electrical properties. Strong interactions between the inorganic and organic units can lead to novel or improved physical or chemical performance relative to that of the individual components, realizing synergistic (" $1+1>2$ ") performance. It is of great scientific significance for the development of basic scientific research: Understanding and interpretation the law of molecular self-assemble, controlling the self-assemble of low dimensional molecular aggregation with high ordered degree in large area through tailoring the molecular structure and the interaction forces, understanding the synergy drive mechanism produced by the weak interactions between the molecular aggregations then optimizing the original function through the hybrid/ heterojunction self-assemble. In this paper, we discuss the synthetic methods for preparing heterojunctions incorporating diverse components and their potential applications in the fields of electronics and optics.
\end{abstract}

one dimension nanomaterial, heterojunction, AAO template

Citation: Qian X M, Liu H B, Li Y L. Self-assembly low dimensional inorganic/organic heterojunction nanomaterials. Chin Sci Bull, 2013, 58: 2686-2697, doi: $10.1007 / \mathrm{s} 11434-013-5881-4$

From the 1980 s to the early 1990 s, self-assembly technology has a rapid development in the world which makes the small scale multidisciplinary cross showing great vitality and becoming a widely subject content with potential application in the field of fundamental research. Heterogeneous structured, composite, low dimensional and intelligence are the development trend and urgent need of modern materials in the fields of photoelectric and information technology. Combining different molecules with different structures, components and functions through self-assemble technology could complement, optimize and collaboratively enhance the basic characteristics of materials. At the same time, the research on complex systems can lead to new concept, new features and new materials which representing the development directions of current self-assembly technology and materials research. Heterojunction material is

*Corresponding author (email: ylli@iccas.ac.cn) referred to a kind of composite material which combining two or more components and forming one or more well-knit interfaces. So far, the heterojunctions can be roughly divided into the following categories: metal-metal junctions [1], metal-polymer junctions [2], inorganic-inorganic junctions [3], inorganic-carbon nanotube junctions [4], inorganicmetal junctions [5], inorganic-organic semiconductor junctions [6-11]. Recently, the research about organic functional molecules in the state of aggregation structure has got rapid development. But, whether inorganic or organic functional materials, as a single material, it is difficult to satisfy various needs of the development of multifunctional materials. Combining inorganic and organic materials in a covalent or non-covalent manner to form one or more interfaces, the properties of these new functional materials arise from the sum of the contributions of the individual components as well as from processes that occur at the interfaces between the components, thereby realizing synergistic 
performance (" $1+2>2$ ") performance.

The main research object, molecular low dimensional state of aggregation structure in nanometer scale, is in a certain dimension size less than $100 \mathrm{~nm}$. Such nanomaterials include zero-dimensional (OD) nanoparticles [12,13], one-dimensional (1D) nanowires and naotubes [14-16] and two-dimensional (2D) nanofilms and nanowalls. Onedimensional nanomaterials with lower dimensions and larger aspect ratios make their own unique optical and electrical performance. During the past decades, there is growing considerable attention in such 1D nanostructures because of the diverse functional electronic characteristics and exciting prospects for utilizing these materials in future electronic applications [17-21]. The 1D nanomaterials could be prepared as optoelectronic devices which can be used as field effect transistors [19-21], logic gates [22], light emitting diodes [23-25], solar cells [26-30] and photoelectric detectors [31-33]. And the heterojunction interfaces act as a key role in these devices.

One-dimensional nanoscale heterostructure materials could be prepared using various methods, including chemical vapour deposition (CVD) [34], controlled solid-solid reaction [35], electro-deposition [36], template-assisted synthesis [37] and electro-spinning technique [38].

\section{Self-assembly of inorganic/organic heterojunction nanowires with templates}

One-dimensional heterojunction nanowires could be prepared using various methods, including vapour-phase methods, solution-phase methods, template-directed synthesis, lithography and self-assembly [39]. Template-directed methods have been used widely because they are simple and convenient and provide controllable properties. According to the physical state of the template, these techniques can be classified into hard template and soft template methods. The hard template is typically a thin porous film prepared form materials such as aluminium oxide (AAO), silica, and nanochannel glass. AAO templates are used widely and available commercially in a limited number of pore diameters. There are several methods for transforming materials deposited in the cylindrical pores of templates into nanowires or nanotubes, including pressure injection, vapour deposition, chemical deposition and electro-deposition. The selection of a method for loading the pores is materialdependent, for example, the electro-deposition method requires electro-active materials. The diameters of the resulting nanostructures formed within these templates are determined by the pore diameters. Thus, a major advantage of the hard template method is the ability to control the size and uniformity of the 1D nanostructures. Through adjusting the deposition conditions, two kinds of morphology, nanowire and nanotube, could be formed in the channels of template. Electrochemical deposition method is a very convenient method which could effectively control the structure parameters of the products, especially good at controlling the length of the products. Combining the electrochemical deposition method and template could be very convenient prepared the 1D nanomaterials and the products would have good repeatability. For example, Mirkin and co-workers [40] prepared segmented Au-PPy and Au-PPy$\mathrm{Au}$ rods through electrochemical deposition of gold into the AAO templates and subsequent electrochemical polymerization of pyrrole. Monitoring the current and time that passed during the electrodeposition process allowed control over the length of each block. Using the similar procedure, the Whitesides group also fabricated Au-PANI nanofibers (Figure 1) [41]. They first electrochemically deposited $\mathrm{Au}$ nanotubes, then electrochemically polymerized aniline into the Au nanotubes. At last, they got Au-PANI core/shell nanowires. $\mathrm{Xu}$ and co-workers [42] have also prepared a core/shell structure containing an array of $\mathrm{Ni}$ nanowires enveloped in PANI nanotubes. The initially grown PANI nanotubes acted as a "second-order template" during electrodeposition of the $\mathrm{Ni}$ atoms.

\subsection{Electrodeposition 1D inorganic/organic hetero- junction nanowires using hard template}

The conductive polymers such as PPy and PTH owning high conductivity and characteristics of easy to preparation are chosen to use as p-type organic part in heterojunction nanowires. And the $\mathrm{CdS}$ with strong electric activity characteristics are used as the n-type inorganic part in heterojunction nanowires. After studied the basic electronic structures of PPy and CdS, we found these two types of molecules forming the aggregation states have good matching characteristics. And the CdS/PPy heteojunction nanowires could be conveniently fabricated $[9,43]$. The synthetic procedure is as follows. First, a layer of $\mathrm{Au}$ (about $100 \mathrm{~nm}$ ) was evaporated on one side of the AAO template as a conducting layer, then the AAO template was put into a homemade electrolytic cell as a working electrode with a platinum counter electrode and a saturated calomel electrode (SCE) reference electrode. $\mathrm{CdS}$ nanowires were deposited into the AAO template at a current density of $2.5 \mathrm{~mA} \mathrm{~cm}^{-2}$ in a DMSO solution consisted of $0.55 \mathrm{~mol} / \mathrm{L} \mathrm{CdCl}_{2}$ and 0.19 $\mathrm{mol} / \mathrm{L}$ element sulphur at $130^{\circ} \mathrm{C}$. PPy deposition was carried out from a $0.1 \mathrm{~mol} / \mathrm{L}$ pyrrole and $0.1 \mathrm{~mol} /{\mathrm{L} \mathrm{LiClO}_{4}}$ acetonitrile solution by applying a voltage of $0.85 \mathrm{~V}$ (vs. SCE) for an appropriate time. Finally, the AAO template was selectively etched by $\mathrm{NaOH}$ solution $(3 \mathrm{~mol} / \mathrm{L})$ and cleaned by deionized water for latter analysis. The SEM image (Figure 2(a)) shows there are a lot of nanowires arranged together. And the more bright part is $\mathrm{CdS}$, the gray part is PPy nanowires. Under the larger magnification, CdS and PPy nanowire attaches to form a clear interface. The results of elements linear scanning show there are a lot of carbon element uniform distributing in the left side of the 

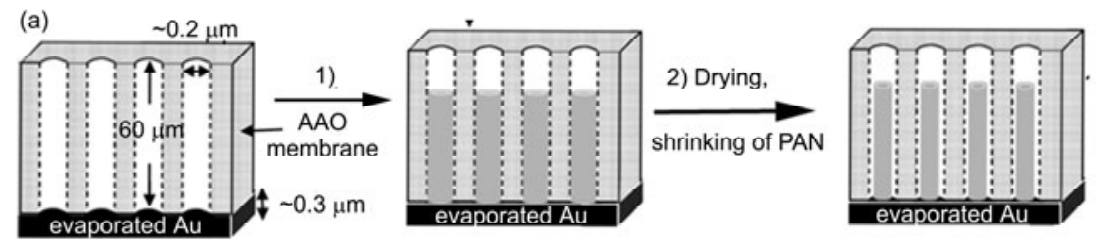

1) Aniline electropolymerization

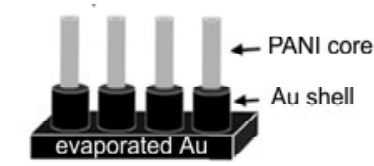

4) Complete dissolution of membrane in $1 \mathrm{~mol} \mathrm{~L}^{-1} \mathrm{NaOH}$

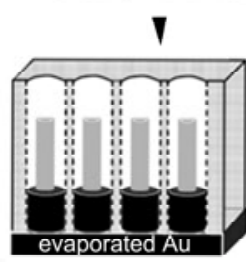

3) Audeposition ( $\mathrm{pH}$ 10.2), partial dissolution of membrane walls
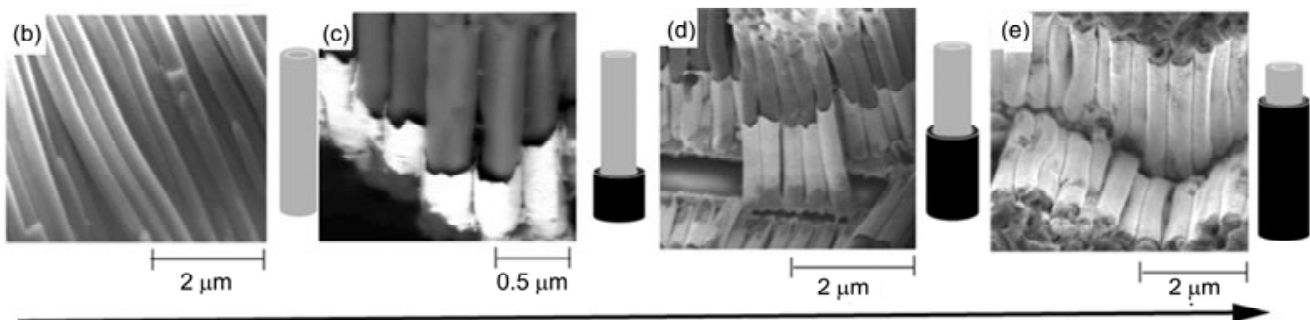

Increasing Au Electrodeposition time (charge)

Figure 1 Procedure for the preparation of the segmented polymer-metal nanostructures within the pores of a Cu-backed AAO membrane (a); SEM images of PANI fiber only (b); PANI-Au core/shell structures where the Au was deposited for $1 \mathrm{~h}$ (c), $1.5 \mathrm{~h}$ (d), and $2.5 \mathrm{~h}$ (e). Reproduced with permission from the American Chemical Society, ref. [41].
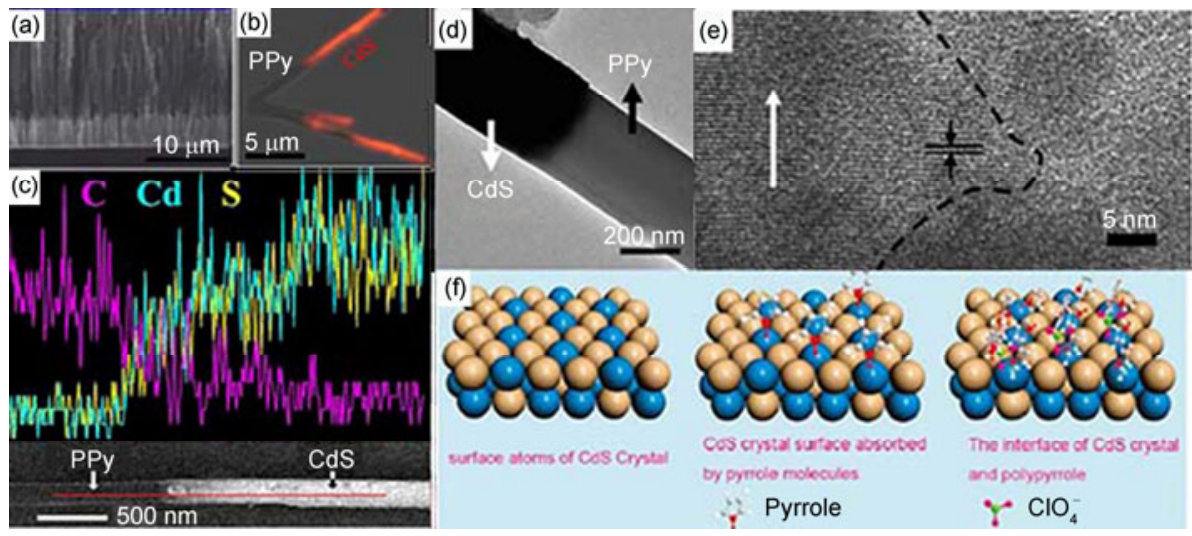

Figure 2 (a) The side view SEM image of CdS/PPy nanowires; (b) the microscope image of single CdS/PPy nanowires under $405 \mathrm{~nm}$ laser; (c) the element linear scanning image; (d) low magnification TEM image; (e) the HRTEM image; (f) models of the surface of CdS in different growing stages. Reproduced with permission from the American Chemical Society, refs. [9,43].

interface and a lot of cadmium element uniform distributing in the right side of the interface (Figure 2(c)). The CdS part of the single nanowire shows red fluorescence because its surface-trap emission ranged from 570 to $670 \mathrm{~nm}$ under excitation of $405 \mathrm{~nm}$ laser (Figure 2(b)). The low magnification TEM image shows (Figure 2(d)) CdS and PPy nanowire combines tightly to form a flat interface without obvious defects. The HRTEM image shows the polycrystalline CdS attached firmly with amorphous PPy. The lattice spacing around $0.34 \mathrm{~nm}$ observed in the image agrees well with interplanar distance of the (002) direction parallel in the hexagonal wurtzite phase of CdS. Then, it could be concluded the growth process of CdS/PPy nanowires. First of all, CdS nanowires were deposited into the pour of AAO template and gradually filled part of the channel. When the pyrrole solution was injected into the electrolytic cell, the top side of CdS nanowires started absorption pyrrole molecules. Because of the $-\mathrm{NH}$ group and $\mathrm{Cd}^{2+}$ having certain coordination effect, CdS and pyrrole molecules can be connected together firmly. After applied $0.85 \mathrm{~V}$ voltage, pyrrole began to polymerize and grow on the top side of $\mathrm{CdS}$ nanowires. Meanwhile, the $\mathrm{ClO}_{4}{ }^{-}$group as the electrolyte of lithium perchlorate were doped into the polypyrrole molecular fragments. The heterojunction formed by the interaction among $\mathrm{Cd}^{2+},-\mathrm{NH}$ and $\mathrm{ClO}_{4}^{-}$was so strong that it could not be broken even under the ultrasonic. 
Increase the heterogeneous circle area can greatly improve the performance of the heterojunction. During the process of preparation of conductive polymer, it was found that there are small gaps between polymer nanowires and template channels. If these gaps could be filled by $\mathrm{CdS}$ which would greatly increase the area of the interface.

Method to prepare PTH/CdS heterojunction nanowires was developed (Figure 3) [10]. The PTH nanowires were first deposited into the AAO template then synthesized $\mathrm{CdS}$ nanowries under two different current densities, 2.5 and 5 $\mathrm{mA} \mathrm{cm}{ }^{-2}$. The Figure 4(a) shows SEM image of PTH/CdS nanowries in which the $\mathrm{CdS}$ nanowries were synthesized under small current density. The top part of PTH nanowires is wrapped around CdS forming core/shell nanostructure. But there is no such phenomenon in the PTH/CdS nanowires which were synthesized under large current density. The key factor leading to this phenomenon lies in controlling the growth speed of $\mathrm{CdS}$ nanowries. Under the small deposition current density, the CdS nanocrystals precipitating from the reaction solution were small which could help for their deposition into the gaps between PTH nanowire and template channel, then formed the core/shell nanostructures. Under the larger current density, the CdS nanocrystals were too large to embed the gaps. There is no fluorescence when PPy, PTH and PANI excited by light which makes some shortage in the optical performance of inorganic/organic heterojunction nanowires. So, we designed a new polymer which can emit fluorescence at the same excitation light with different colour. 1,4-Bis(pyrrol-2-yl) benzene (BPB) was chosen as the polymer monomer [44] and PBPB/CdS heterojunction nanowires were synthesized. When the nanowires were excited by mercury lamp with wavelength range 330 to $380 \mathrm{~nm}$, both ends of the naowires issued the fluorescent with different color. The part with red fluorescence is $\mathrm{CdS}$ and the other part with yellow green fluorescent part is PBPB. The preparation methods about organic polymer heterojunction nanowires were also studied (Figure 5) [45]. The PEDOT/PT34bT copolymer nanowires were synthesized under the applied voltage $(1.1 \mathrm{~V})$ with an acetonitrile solvent containing $5 \mathrm{mmol} / \mathrm{L}$ EDOT and T34bT monomer.

\subsection{Combining hard template and pressure injection method to construct 1D heterojunction nanowires}

The combination of pressure injection with template method

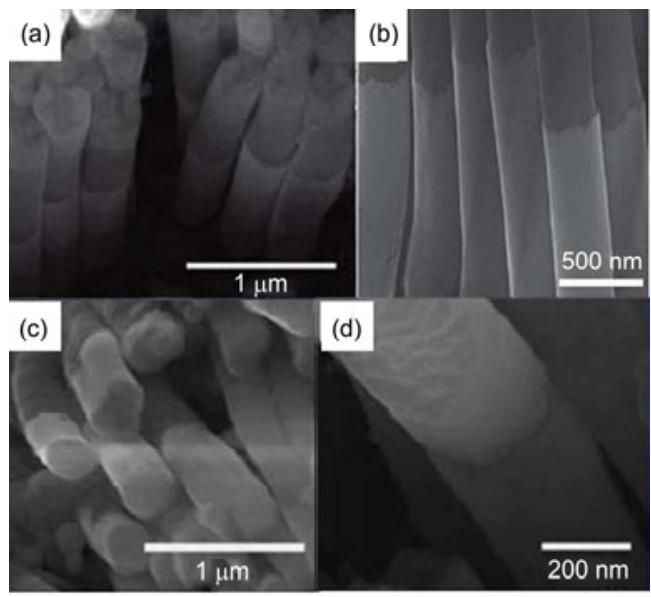

Figure 3 The SEM images of CdS/PTH core/shell nanowire arrays (a) low magnification image; (b) large magnification image; the SEM images of CdS/PTH segmented nanorods; (c) low magnification image; (d) large magnification image. Reproduced with permission from the American Chemical Society, ref. [10].

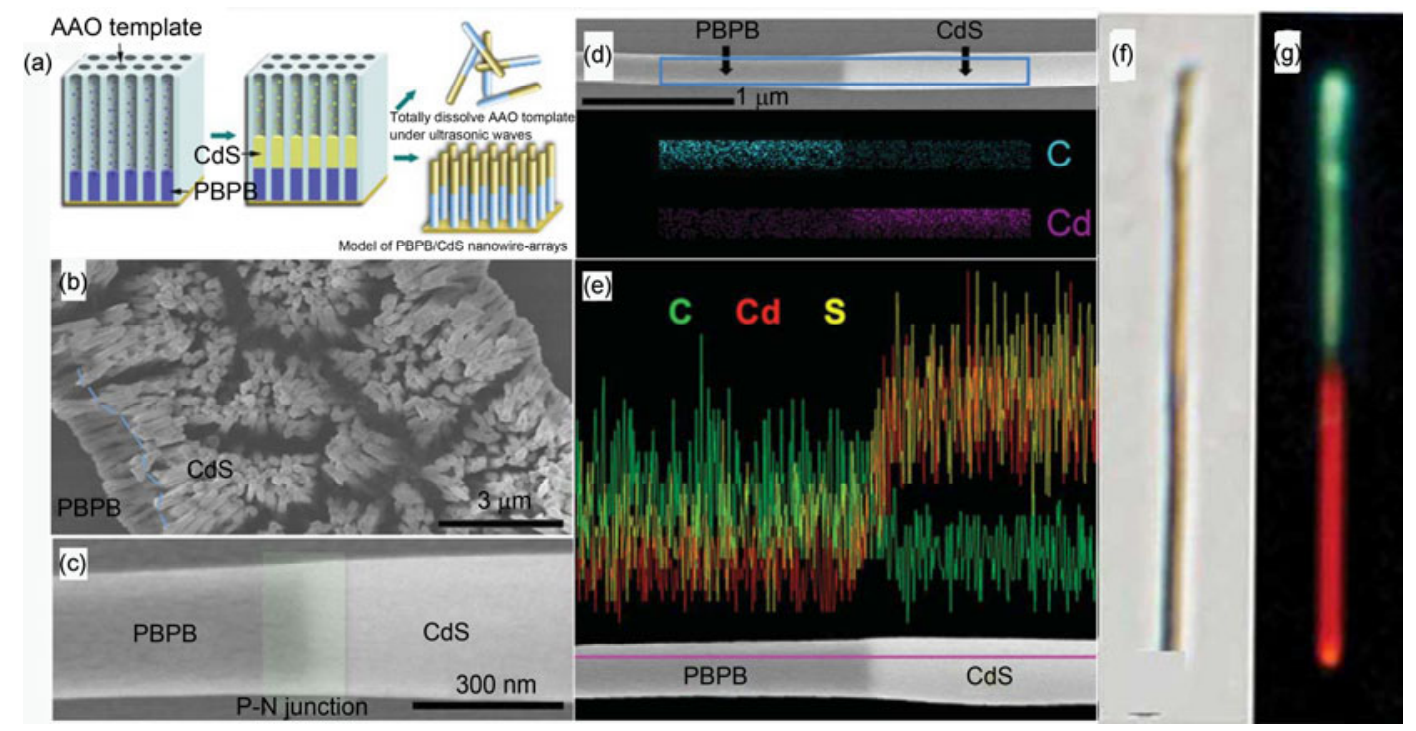

Figure 4 (a) The scheme of preparation of CdS/PBPB nanowires; (b) the SEM image of CdS/PBPB nanowires; (c) the SEM image of single CdS/PBPB nanowire under larger magnification; (d) and (e) the element surface and linear scanning images of single CdS/PBPB nanowire; (f) and (g) the microscope images of single CdS/PBPB naowire under white light and mercury lamp. Reproduced with permission from the Royal Society of Chemistry, ref. [44]. 

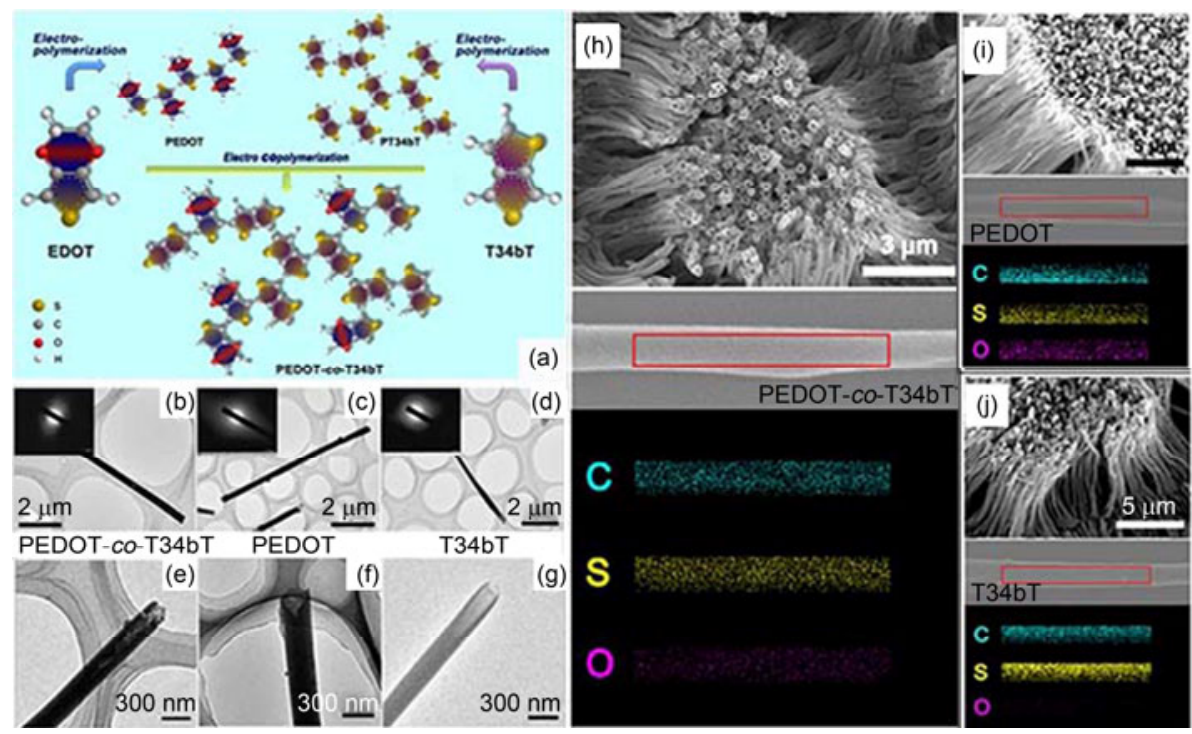

Figure 5 (a) The scheme of preparing PEDOT, PT34bT, PEDOT and PT34bT copolymerization nanowires; (b)-(g) the TEM images of nanowires; (h) the SEM and element surface scanning images of PEDOT-co-T34bT nanowires; (i) the SEM and element surface scanning images of PEDOT nanowires; (j) the SEM and element surface scanning images of PT34bT nanowires. Reproduced with permission from the Royal Society of Chemistry, ref. [45].

is another popular approach for the fabrication of $1 \mathrm{D}$ nanostructures. Template-assisted pressure injection was used to fabricate nanowires comprising organic semiconductors oligo(phenylenevinylenes) (OPVs) and inorganic semiconductors $\mathrm{CdS}$. A porous AAO template was immersed into the transparent CdS-OPV3 mixture in a flask under reduced pressure. The mixture was then exposed to the atmosphere, the solution was forced into the pores of the template as a result of the difference in pressure. The membrane was removed and dried immediately. After repeating these steps three times, we acquired CdS-OPV3 nanowires embedded into the channels of AAO template. The results of EDS analysis, element mapping and confocal laser scanning microscopy analysis indicated the nanowires combined CdS and OPV3 to form the homogeneous hybrid interfaces.

\subsection{Soft template methods}

The generation of heterostructures based on soft templates is rare. Yao and co-workers [46] reported the synthesis of triblock microrods of combining 1,3-dipheny1-2-pyroline (DP) and 4-(dicyanomethylene)-2-methy1-6-(p-dimethylaminostyryl)-4H-pyran (DCM). Figure 6(a) shows the mechanism of formation of the triblock microrods. First, some of the DP molecules were dissolved within the hydrophobic cores of the spherical CTAB micelles to induce the formation of rodlike micelles. These rodlike micelles acted as templates, directing the primary growth of DP particles in one dimension. Finally, a micelle melting process, induced by the micellar sphere-to-rod transition, led to codeposition of the DP and DCM molecules at both ends of (a)

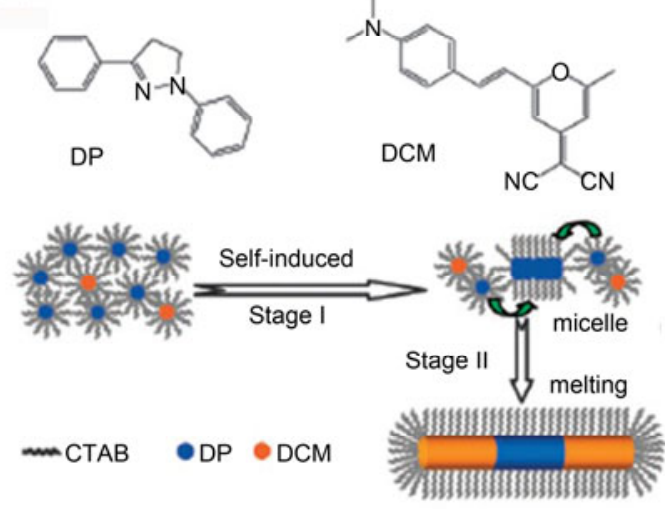

(b)
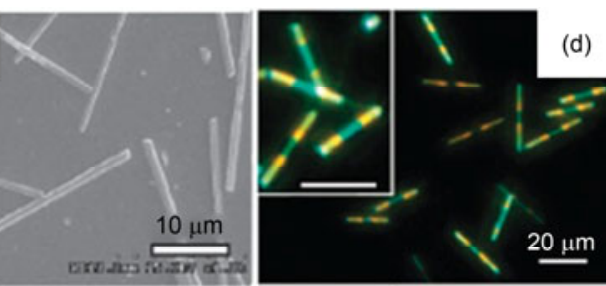

(c)
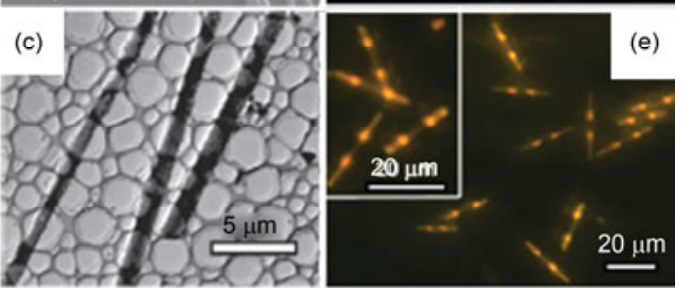

Figure 6 (a) Molecular structures of DP and DCM and the formation mechanism of triblock nicrorods; (b) SEM and (c) TEM images of microrods; fluorescence microscopy images of same microrods excited with unfocused (d) UV (330-380 nm) and (e) blue light (460-490 nm). Reproduced with permission from the American Chemical Society, ref. [46]. 
the as-formed primary OD particles, generating the triblock microrods. Figure 6(b) and (c) revealed smooth outer surface, with each rod having a quite-uniform diameter $(1 \mu \mathrm{m})$ along its length $(20-25 \mu \mathrm{m})$.

\section{Fabrication heterojunction nanowire arrays without templates}

Organic charge transfer complexes are an important class of organic-inorganic hybrid materials, which have unique photo, electrical and photoelectronic properties allowing them to be good candidates for developing nanodevice applications. Under the certain temperature, 7,7,8,8tetracyanoquinodimethane (TCNQ) powder could be gasified and react with cooper film to generate CuTCNQ complexes (Figure 7(a) - (d)) [47-50]. Through controlling the concentrations of TCNQ vapor, two kinds of morphologies of CuTCNQ complexes, nanowire and nanotube, could be fabricated (Figure 7(e) and (f)) [51]. And the concentration of TCNQ vapor could be adjusted by controlling the heating rate. In a process of slow heating, TCNQ powder will continue to evaporate out of the steam with low concentration. This steam will react quickly with copper to generate amounts of small CuTCNQ complexes particles depositing on the surface of copper as the seeds growing points. Meanwhile, the gas-solid interface has enough sources to supply copper ions. So the CuTCNQ complex will grow priority on these growing point and form whole nanowire. If rapid heating the organic source, TCNQ powder will volatilize quickly in a short time to form a high concentration steam and then forming a large number of seeds depositing on the copper film. In this time, the concentration of copper ions on the gas-solid interface dropped rapidly. So the out wall of the CuTCNQ complex could grow priority and then form nanotube structure.

5,10,15,20-[1,4-benzodioxane-6-carboxalde]porphyrin (TEOP) was used as raw material and to prepare 0D nanoballs through self-assemble method [52]. In the whole selfassemble process, the environment and solution temperatures

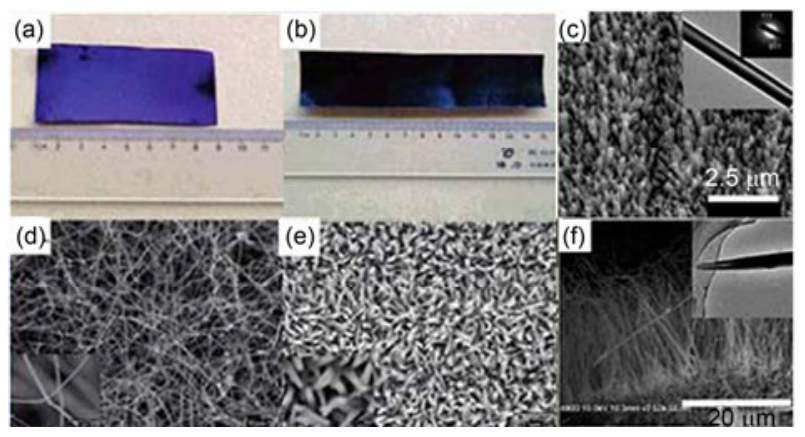

Figure 7 (a) and (c) the photo and SEM image of AgTCNQ nanowires; (b) and (d) the photo and SEM image of CuTCNQ nanowires; (e) and (f) SEM images of CuTCNQ nanotube arrays. Reproduced with permission from the American Chemical Society, refs. [47,51]. were controlled at 15 and $25^{\circ} \mathrm{C}$. The assemble process is as follows. First, using $10 \mu \mathrm{L}$ TEOP chloroform solution $\left(10^{-3}\right.$ $\mathrm{mol} / \mathrm{L})$ to drop on the silicon surface, and then using $11 \mu \mathrm{L}$ isopropyl alcohol and water (v/v, 10:1) mixed solvent to inject into TEOP solution. The morphology of TEOP could be adjusted by controlling the solvent evaporation time. The forming process of TEOP nanoballs regularly arranged was determined by the diffusion and evaporation rate of the mixed solvent. When the poor solvent of PEOT, isopropyl alcohol, was injected into the chloroform, two kinds of solvents had mutual diffusion. Then, the TEOP molecules were precipitated form the mixed solvent forming J-type aggregates. These aggregates started layer upon layer to form nanoball structures through the $\pi-\pi$ effect between porphyrin molecules. When the solvent was heated by constant temperature, TEOP molecules will continue to precipitate from the solvent. Then, the number and volume of nanoballs will continue to increase. And the heating process could enhance the convection of the solvent which could increase the probability of collision and aggregation between nanoballs. With increase the evaporation time, in some areas on the surface of wafer will appear nanoballs arranging into line (Figure $8(n)$ ) which will be a template to induce nanoball forming ordered arrangement in some small areas (Figure 8(o)). As a result, the area of the ordered arrays was extended (Figure 8(r)).

\section{Fabrications and applications of 1D inor- ganic/organic heterojunction nanomaterials}

\subsection{Diodes}

Diodes, devices that control current flow in one direction, are usually employed as switches in electrical circuits. The CdS/PPY heterojunction nanowires show good optical diode characteristic (Figure 9) [43]. Without light, CdS nanowires are almost of the insulation. But with the increase of illumination, the conductivity of $\mathrm{CdS}$ nanowires is improved gradually. The PPY nanowires also show some photoelectric response characteristics. Without illumination, the CdS/PPY nanowires did not show diode rectifier features. But under the light, the heterojunction nanowires showed obvious effects of diode rectifier, and with the increase of illumination intensity the rectification ratio also increased. When applied $5 \mathrm{~V}$ forward biased and adjusted light intensity from 1.12 to $5.76 \mathrm{~mW} \mathrm{~cm}^{-2}$, the rectification ratio increase from 8 to 13 . It is just the heterojunction effect causing this peculiar photoelectric response characteristic which will have an important guiding role to the application of nanometer circuit and devices [44]. In order to get the upright $\mathrm{PBPB} / \mathrm{CdS}$ heterojunction nanowire arrays, the template was corroded off without polished off the gold film which direct contacted with $\mathrm{PBPB} / \mathrm{CdS}$ nanowires as the bottom electrode (Figure 10(a)). Using ITO glass as the top electrode contacted with $\mathrm{CdS}$ nanowires. Applied 30 

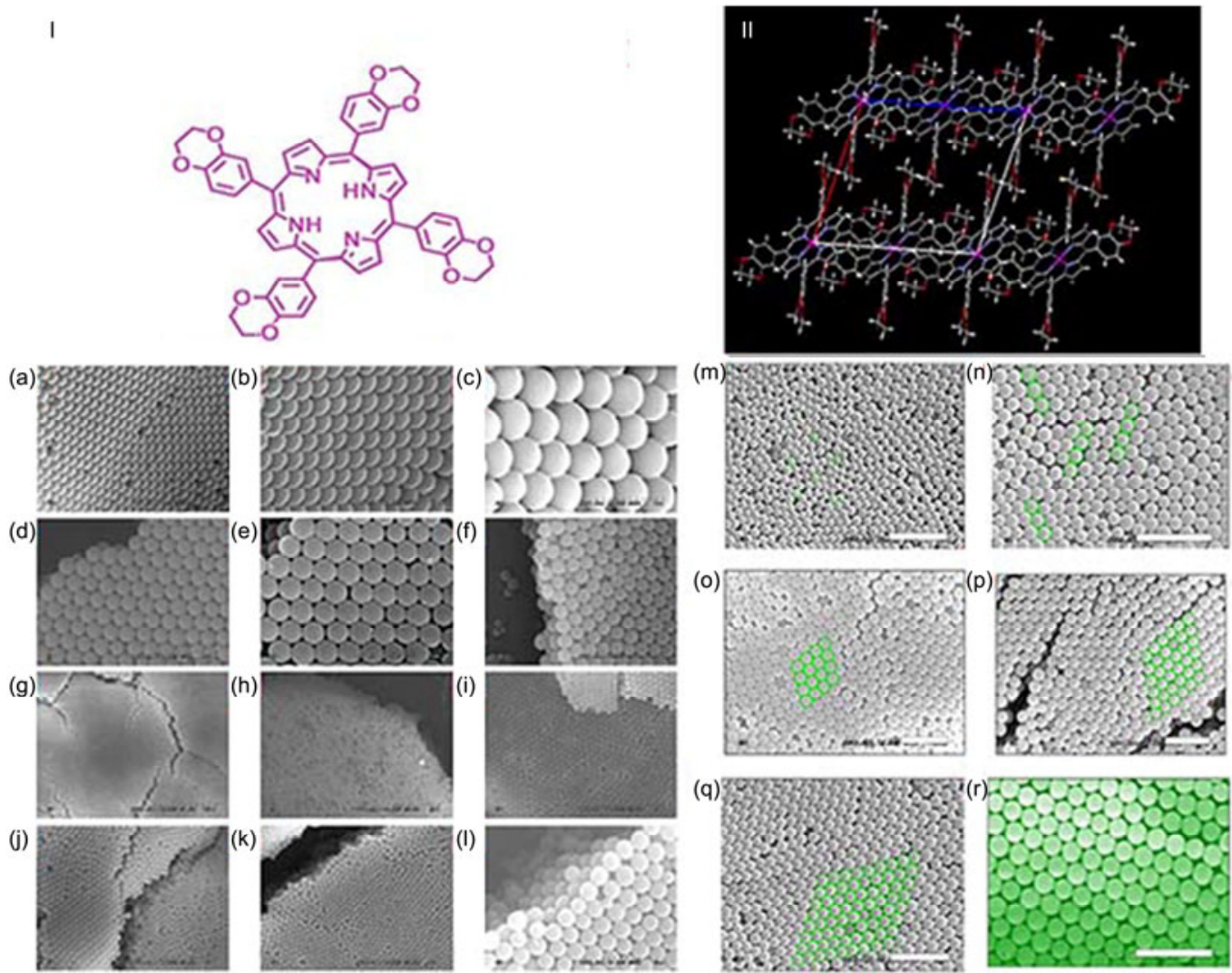

Figure 8 (I) The molecular structure of TEOP; (II) the crystal structure of TEOP; (a)-(c) single layer film; (d) double membrane; (e) three layers film; (f) four layers film; (g)-(l) multilayer film; (m) -(r) TEOP pattern prepared by thermal treatment in 5, 6, 7, 8, 9 and 10 min [52].
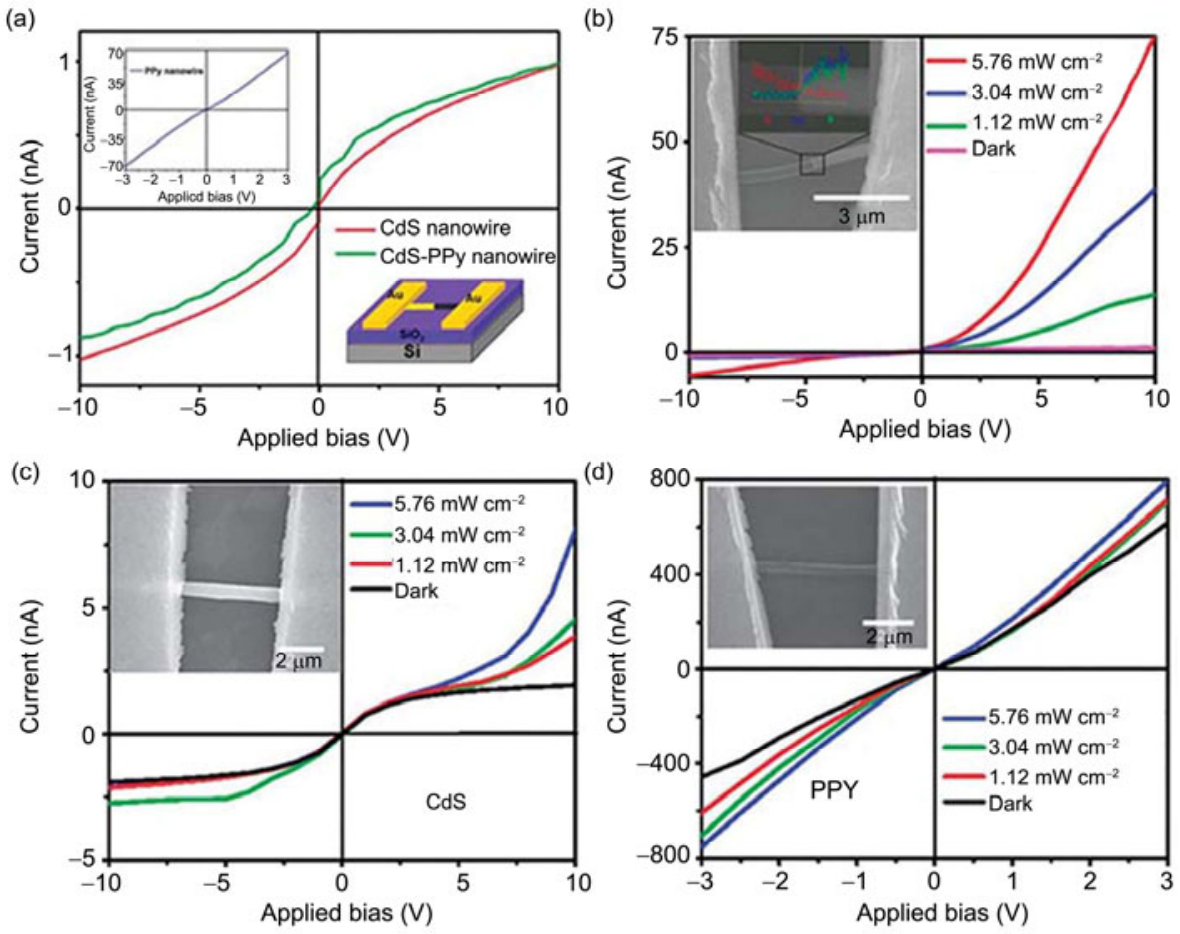

Figure 9 (a) The IV curves of single CdS/PPY nanowire without light; (b) the IV curves of single CdS/PPY nanowire under different illumination intensity; (c) and (d) the IV curves of single CdS and PPY nanowire under different illumination intensity. Reproduced with permission from the American Chemical Society, ref. [43]. 

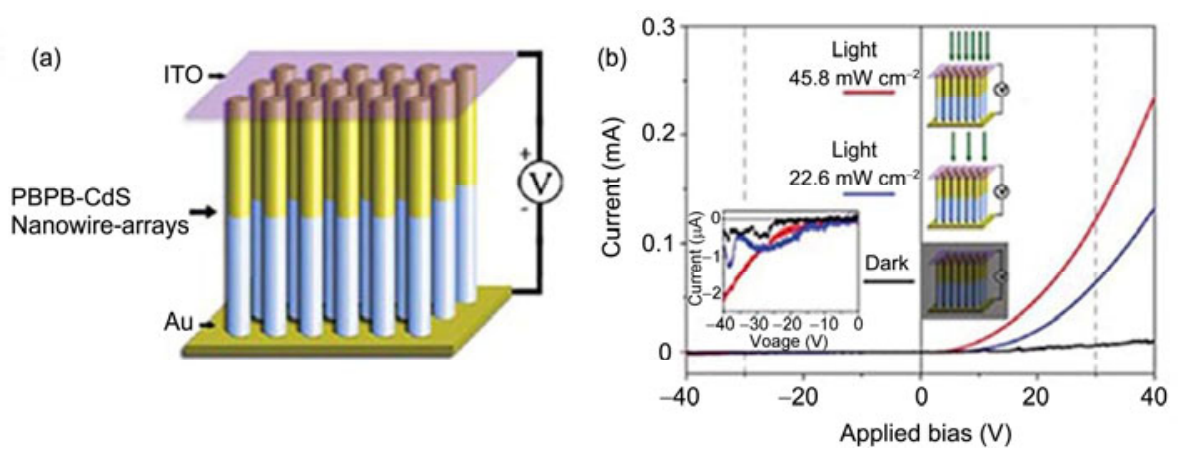

Figure 10 (a) The scheme of CdS/PBPB nanowire arrays device; (b) the IV curves of CdS/PBPB device under different illumination intensity. Reproduced with permission from the Royal Society of Chemistry, ref. [44].

bias and with three different illumination intensity $(0,22.6$ and $45.8 \mathrm{~mW} \mathrm{~cm}^{-2}$ ), the rectification ratio of heterojunction nanotube arrays are 29.9, 116.2 and 129.7. The heterojunction nanowires containing $\mathrm{CdS}$ ingredients showed a great resistance without light and it is conductivity would be improved by increasing the illumination intensity. Investigating its reason, the results should be attributed to the light sensitivity of $\mathrm{CdS}$ and the good contact between $\mathrm{CdS}$ and organic materials. It was speculated that the photoelectric response characteristics of CdS must have certain selectivity. So, CdS/PANI heterojunction nanowires were fabricated to investigate the selectivity characteristics with different wavelength light source [7]. There are two purposes to replace PBPB with PANI. First, PANI has not light response which will not interfere with the experiment results; second, the conductivity of PANI is far worse than PBPB which will increase the sensitivity of the experiment. The Figure 11(a) shows the gold film as bottom electrode contacts the PANI nanowires and the ITO glass as top electrode contacts with $\mathrm{CdS}$ nanowires. Without light, the device shows a great resistance. Then, we chose 17 different wavelength of light source (from 254 to $610 \mathrm{~nm}$ ) in the same illumination intensity to irradiate the sample and observe its optical diode rectifier features. Figure 11(b) shows the sample has the largest rectification ratio under $420 \mathrm{~nm}$ light, indicating the heterojunction nanowires with very high selectivity. Through adjusting the blue light intensity, the relationship between rectification ratio and illumination was gotten which could be expressed by a linear formula: $R=4.13 E+$ 11.67 , where the $R$ is rectification ratio, $E$ is the illumination intensity. Using this formula could sensor the blue light intensity by CdS/PANI device.

\subsection{Photovoltaic performance}

The inorganic/organic hybrid thin film solar cells have gained great attention because they are inexpensive, easily solution-processed and highly stable. Several studies on the photovoltaic properties of nanowires, especially a single nanowire of semiconductor with $\mathrm{p}-\mathrm{n}$ junction, have been described recently. Liber and co-workers [53] prepared photovoltaic devices incorporating single coaxial p-type/ intrinsic/n-type ( $\mathrm{p}-\mathrm{i}-\mathrm{n})$ silicon nanowires. Under simulated one-sun irradiation, the optimized p-i-n SiNW devices exhibited an open-circuit voltage of $0.29 \mathrm{~V}$, a maximum short-circuit current density of $3.5 \mathrm{~mA} \mathrm{~cm}{ }^{-2}$, and a maximum efficiency of $0.5 \%$. Single CdS/PPY heterojunction nanowire photovoltaic device was fabricated and tested its photovoltaic properties (Figure 12) [9]. Under the illumination, $\mathrm{CdS}$ nanowires product light carrier, holes and electrons, which are quickly separated near the junction interface and reach the gold electrodes. The CdS/PPY nanowires have wide absorption spectrum which helps the device to work in the visible light and improve the photoelectric conversion efficiency. The biggest photoelectric conversion efficiency reached $0.018 \%$ under illumination intensity of $6.05 \mathrm{~mW} \mathrm{~cm}^{-2}$.

\subsection{Field emission properties}

Low voltage electronic field emission has attracted much attention because of its possible application in microwave power amplification, space propulsion, e-beam lithography and display devices. Larger area of CuTCNQ and AgTCNQ nanowire arrays were fabricated to investigate their field emission performance [47], and their turn on field are about

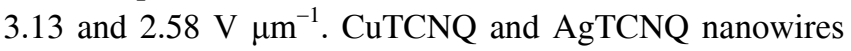
are very promising field emission cathode materials which have smaller work function, even than carbon materials such as graphene. Through changing the growth condition, the CuTCNQ nanowall [49] and nanotube [51] arrays were also fabricated. Their turn on fields were 8 and $12.2 \mathrm{~V} \mathrm{\mu m}^{-1}$ and the largest emission current density were 10.9 and 7.15 $\mathrm{mA} \mathrm{cm} \mathrm{cm}^{-2}$.

Through adjusting growth conditions, CdS/PTH segment nanorods and core/shell nanowires were fabricated and they showed good field emission characteristics. Figure 13 [10] shows the CdS/PTH core/shell nanowires have the best field emission properties which is due to its special structure, that is, the CdS shell could increase the area of electron emission. The field emission properties of PEDOT-co-T34bT, PEDOT and PT34bT nanowires were also investigated [45]. 

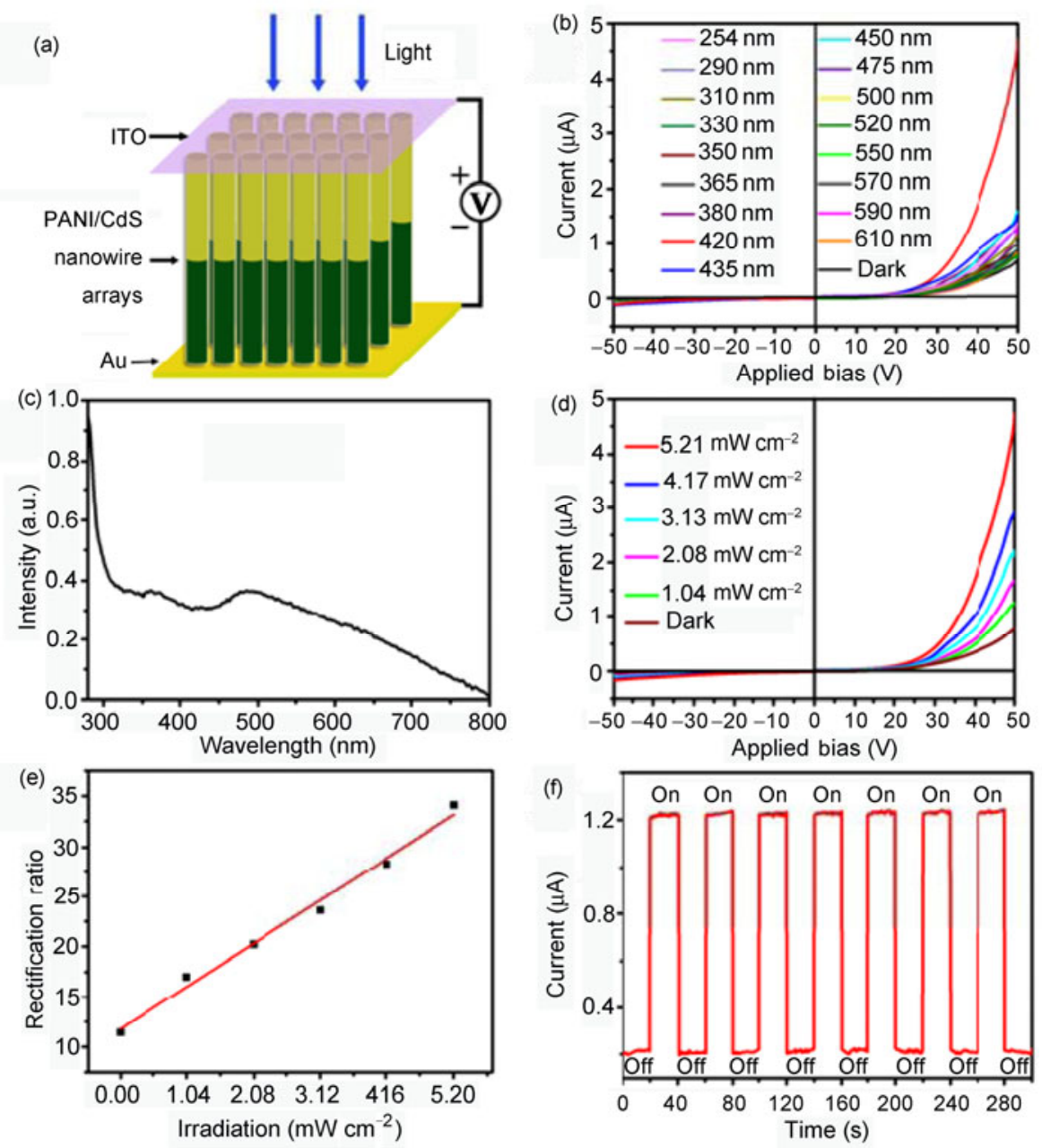

Figure 11 (a) The scheme of CdS/PANI nanowire arrays device; (b) the IV curves of CdS/PANI device under different illumination intensity; (c) the UV pattern of CdS/PANI nanowires; (d) the IV curve of CdS/PANI device under $420 \mathrm{~nm}$ blue light with different intensity; (e) the experimental curve of rectifier with light intensity; (f) the cycle response curve of CdS/PANI nanowires under blgyue light. Reproduced with permission from the American Chemical Society, ref. [7].

Figure 14 shows the turn on field of three samples as about $1.83,3.17$ and $3.83 \mathrm{~V} \mathrm{\mu m}^{-1}$, the threshold field are about

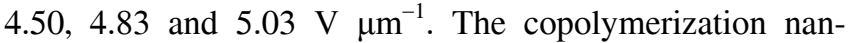
owires have the best field emission performance which should be due to its high conductivity. In the process of electron emission, the ability of electronic transmission will directly affect the field emission properties. And the Figure 14 (d) shows that the copolymerization nanowires have the best conductivity.

\section{Conclusions}

Templates were used to fabricate 1D inorganic/organic heterojunction nanostructure materials, the photoelectric performance of single nanowire was investigated, and large area of metal-organic charge transfer complex were fabricated to study their field emission properties. With the aid of AAO templates, the n-type semiconductor $\mathrm{CdS}$ as inorganic material was assembled with a series of p-type organic polymers to form heterojunction nanomaterials. $\mathrm{CdS} / \mathrm{PPY}$ nanowires were fabricated and investigated their photoelectric performance, which opened a new development direction for the application of nanoscale devices. Through assembling of CdS with novel organic polymers, the optical properties of heterojunction nanowires were improved and controlled. By investigating the response selectivity of the heterojunction nanowires assembled from $\mathrm{CdS}$ and polymers to different wavelength light, good selectivity of heterojunction to the light was found, which was applied to construct blue light detector. The larger area CuTCNQ films were fabricated using organic gas-solid phase reaction in the absence of template and three kinds of morphologies such as nanowires, nanowalls and nanotubes could be obtained through adjusting the experimental parameters. The CuTCNQ nanostructure arrays show lower turn on field and large emission current density which indicate a potential application in field emission devices. 

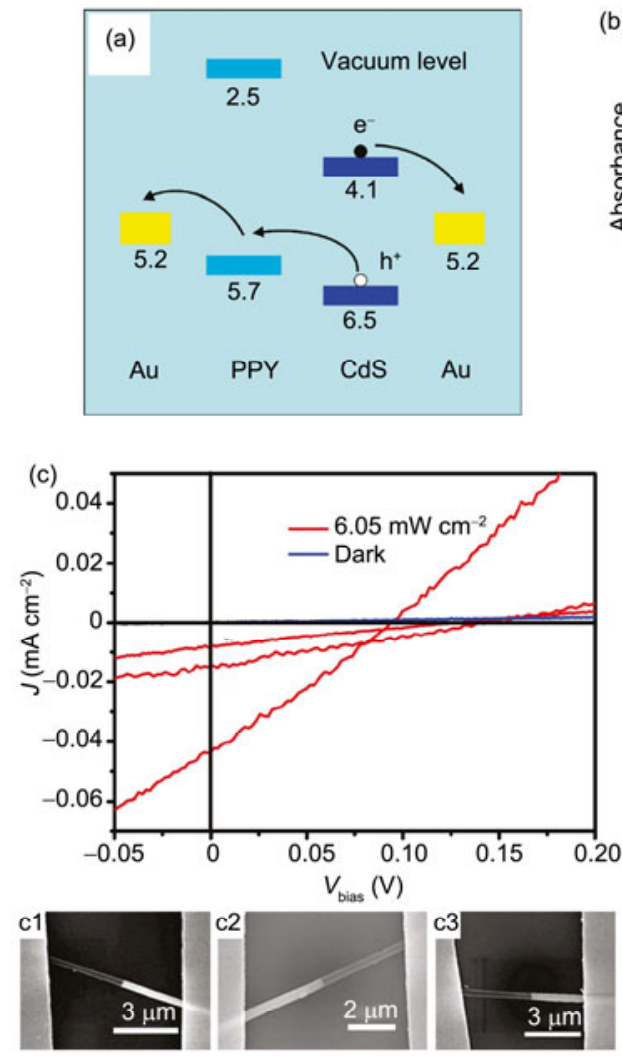
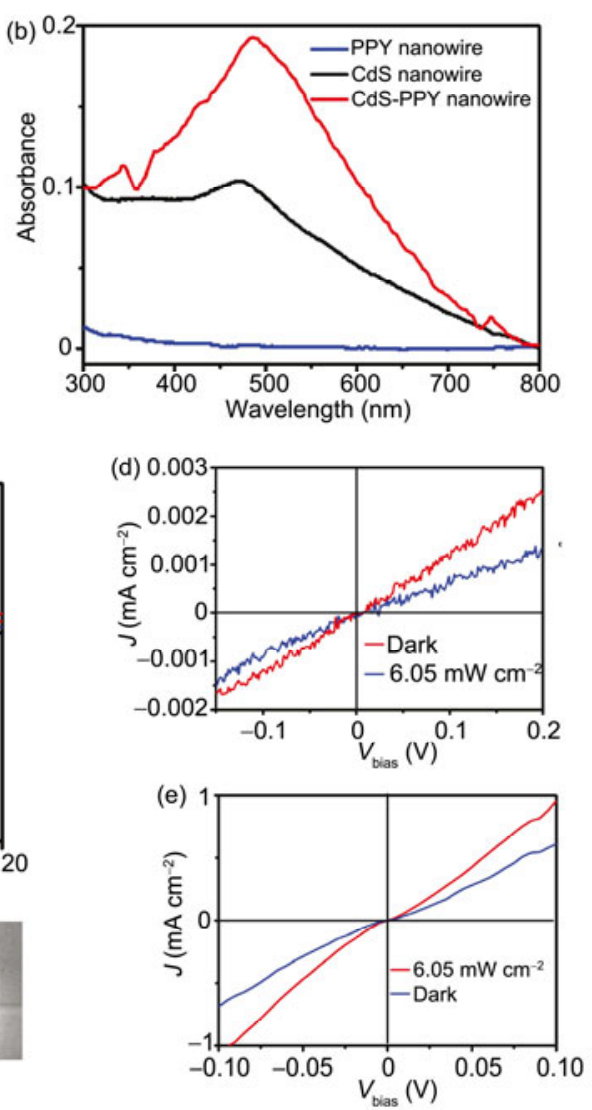

Figure 12 (a) The band gap scheme of CdS/PPY nanowire; (b) the UV patterns of CdS, PPY and CdS/PPY nanowires; (c) the IV curves of CdS/PPY single nanowire devices under different illumination intensity; the length of the single nanowire (c1) 7.428, (c2) 6.922 and (c3) 6.597; (d), (e) the IV curves of CdS and PPY nanowires. Reproduced with permission from the American Chemical Society, ref. [9].
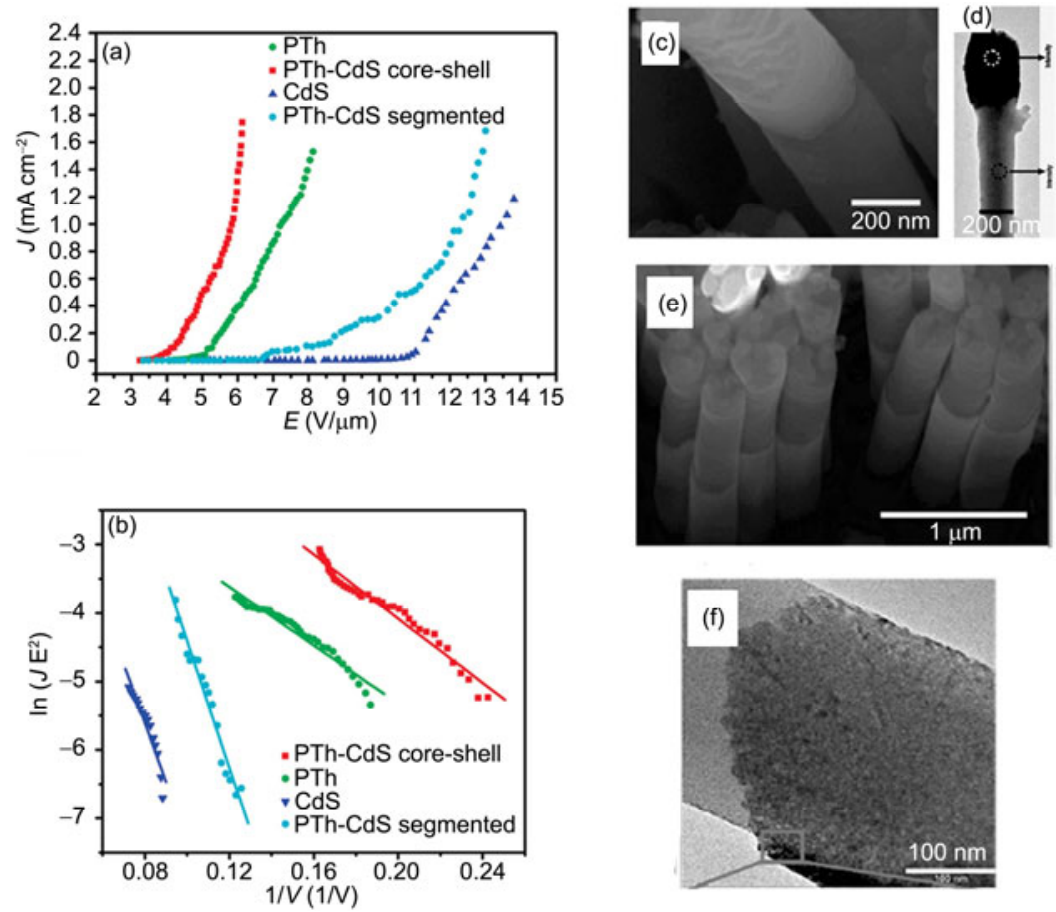

Figure 13 (a) The $J-E$ curves of CdS/PTH nanowire arrays; (b) the corresponding FN curves; (c) the SEM and TEM images of CdS/PTH segment nanorods; (d) the SEM and TEM images of CdS/PTH core/shell nanowires. (e) and (f) SEM and TEM images of CdS/PTH core/shell nanowires. Reproduced with permission from the American Chemical Society, ref. [10]. 

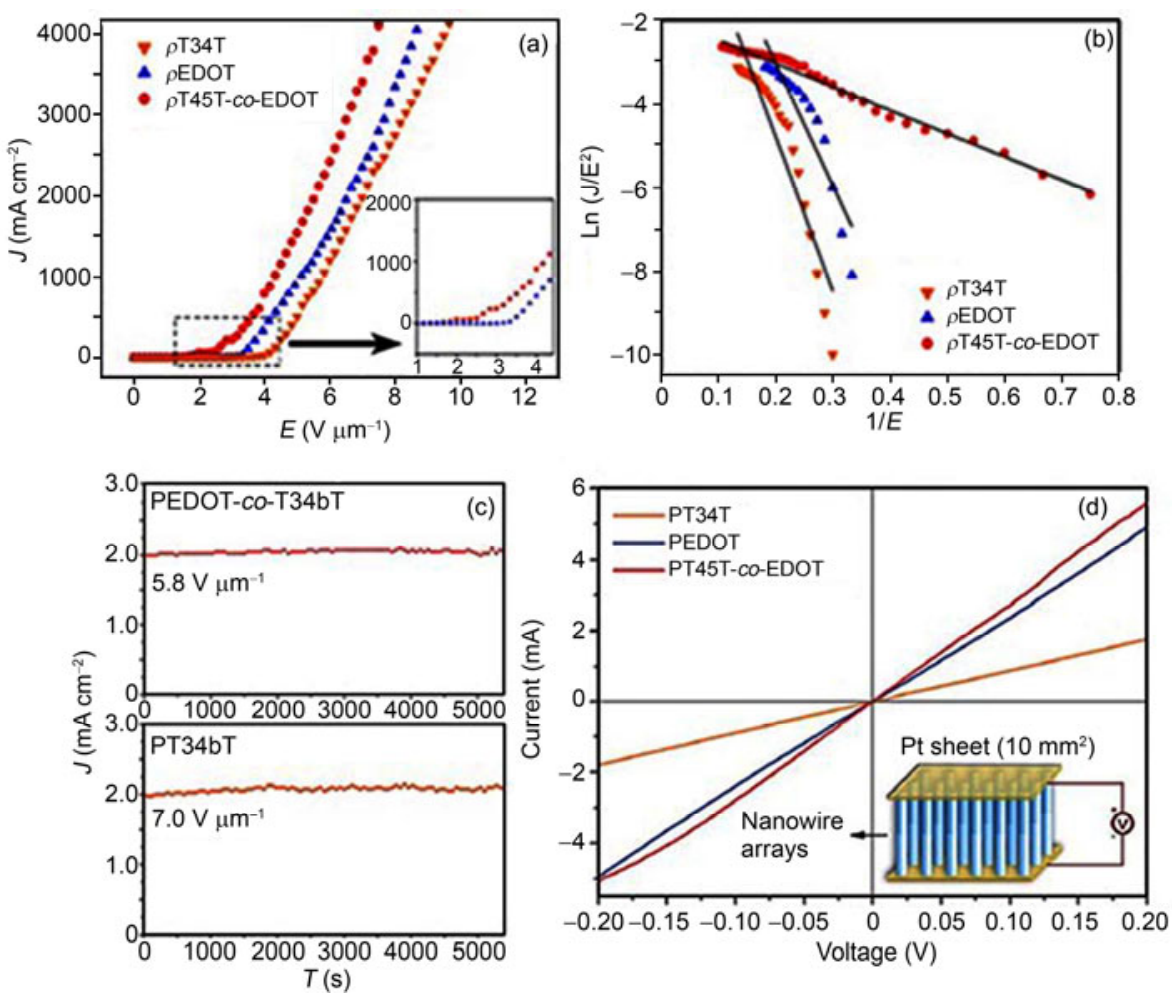

Figure 14 (a) The $J-E$ curves of three samples; (b) the corresponding FN curves; (c) the stability curves of PEDOT-co-T34bT and PT34bT nanowires under different emission current density; (d) the IV curves of three samples. Reproduced with permission from the Royal Chemistry Society, ref. [45].

Inorganic/organic heterojunction nanostructures have multi-function features which render them a great application potential. However, there are three bottlenecks need to be solved for the further development of the heterojunction nanostructures. (1) Methods should be developed to increase the area of interface, improve the ordered degree of self-assemble and to solve the problem of large area and large-scale preparation of the materials. (2) To strengthen the characterization and the investigation of the intrinsic properties of the single nanowire, especial the morphologies and size of the interface. (3) To obtain the heterojunction arrays with more stable structure and performance through controlling the heterojunction's structure, size and interface linkage.

This work was supported by the National Natural Science Foundation of China (21031006) and the National Basic Research Program of China (2011CB932302, 2012CB932900).

1 Liu K, Nagodawithana K, Searson P C, et al. Perpendicular giant magnetoresistance of multilayered $\mathrm{Co} / \mathrm{Cu}$ nanowires. Phy Rev $\mathrm{B}$, 1995, 51: 7381-7384

2 Bognitzki M, Hou H, Ishaque M, et al. Polymer, metal, and hybrid nano- and mesotubes by coating degradable polymer template fibers (TUFT Process). Adv Mater, 2000, 12: 637-640

3 Kovtyukhova N I, Kelley B K, Mallouk T E. Coaxially gated in-wire thin-film transistors made by template assembly. J Am Chem Soc, 2004, 126: 12738-12739

4 Robel I, Bunker B A, Kamat P V. Single-walled carbon nanotube-CdS nanocomposites as light-harvesting assemblies: Photoin- duced charge-transfer interactions. Adv Mater, 2005, 17: 2458-2463

5 Wang W, Song L P, Ryou J H, et al. Growth of uniformly aligned $\mathrm{ZnO}$ nanowire heterojunction arrays on $\mathrm{GaN}$, AlN, and $\mathrm{A} 10.5 \mathrm{Ga} 0.5 \mathrm{~N}$ substrates. J Am Chem Soc, 2005, 127: 7920-7923

6 Lin H, Liu H, Qian X, et al. Controlling the growth of low dimension nanostructures of an iridium complex. Dalton Trans, 2011, 40: 4397-4401

7 Lin H, Liu H, Qian X, et al. Constructing a blue light photodetector on inorganic/organic p-n heterojunction nanowire arrays. Inorg Chem, 2011, 50: 7749-7753

8 Liu X, Li Y. One-dimensional hybrid nanostructures with lightcontrolled properties. Dalton Tran, 2009, 33: 6447-6457

9 Guo Y, Zhang Y, Liu H, et al. Assembled organic/inorganic p-n Junction interface and photovoltaic cell on a single nanowire. J Phy Chem Lett, 2009, 1: 327-330

10 Guo Y, Liu H, Li Y, et al. Controlled core-shell structure for efficiently enhancing field-emission properties of organic-inorganic hybrid nanorods. J Phy Chem C, 2009, 113: 12669-12673

11 Zheng H, Li Y, Liu H, et al. Construction of heterostructure materials toward functionality. Chem Soc Rev, 2011, 40: 4506-4524

12 Alivisatos A P. Semiconductor clusters, nanocrystals, and quantum dots. Science, 1996, 271: 933-937

13 Murray C B, Norris D J, Bawendi M G. Synthesis and characterization of nearly monodisperse $\mathrm{CdE}$ ( $\mathrm{E}=$ sulfur, selenium, tellurium) semiconductor nanocrystallites. J Am Chem Soc, 1993, 115: 87068715

14 Someya T, Werner R, Forchel A, et al. Room temperature lasing at blue wavelengths in gallium nitride microcavities. Science, 1999, 285: 1905-1906

15 Gérard J M, Sermage B, Gayral B, et al. Enhanced spontaneous emission by quantum boxes in a monolithic optical microcavity. Phy Rev Lett, 1998, 81: 1110-1113

16 Tanaka K, Nakamura T, Takamatsu W, et al. Cavity-induced changes of spontaneous emission lifetime in one-dimensional semiconductor microcavities. Phy Rev Lett, 1995, 74: 3380-3383 
17 Wang Z L. Characterizing the structure and properties of individual wire-like nanoentities. Adv Mater, 2000, 12: 1295-1298

18 Hu J, Odom T W, Lieber C M. Chemistry and physics in one dimension: Synthesis and properties of nanowires and nanotubes. Acc Chem Res, 1999, 32: 435-445

19 Duan X, Huang Y, Cui Y, et al. Indium phosphide nanowires as building blocks for nanoscale electronic and optoelectronic devices. Nature, 2001, 409: 66-69

20 Cui Y, Lieber C M. Functional nanoscale electronic devices assembled using silicon nanowire building blocks. Science, 2001, 291: $851-853$

21 Shang-Guan Y G, Chen F, Zheng Q. Microstrucutre, morphology, crystallization and rheological behavior of impact polypropylene copolymer. Sci China Chem, 2012, 5: 698-712

22 Huang Y, Duan X, Wei Q, et al. Directed assembly of onedimensional nanostructures into functional networks. Science, 2001, 291: 630-633

23 Qian F, Li Y, Gradečak S, et al. Gallium nitride-based nanowire radial heterostructures for nanophotonics. Nano Lett, 2004, 4: 19751979

24 Clemens S, Schneller T, van der Hart A, et al. Registered deposition of nanoscale ferroelectric grains by template-controlled growth. Adv Mater, 2005, 17: 1357-1361

25 Huang Y, Duan X, Lieber C M. Nanowires for integrated multicolor nanophotonics. Small, 2005, 1: 142-147

26 Xin H, Kim F S, Jenekhe S A. Highly efficient solar cells based on poly(3-butylthiophene) nanowires. J Am Chem Soc, 2008, 130: 5424-5425

27 Cheung K Y, Yip C T, Djurišić A B, et al. Long K-doped titania and titanate nanowires on Ti foil and FTO/quartz substrates for solar-cell applications. Adv Funct Mater, 2007, 17: 555-562

28 Leschkies K S, Divakar R, Basu J, et al. Photosensitization of $\mathrm{ZnO}$ nanowires with CdSe quantum dots for photovoltaic devices. Nano Lett, 2007, 7: 1793-1798

29 Gong X, Yu T Z, Cao Y, et al. Larger open-circuit voltage polymer solar cells by poly(3-hexylthiophene) with multi-adducts fullerenes. Sci China Chem, 2012, 5: 743-748

30 Yang T B, Qin D H, Lan L F, et al. Inverted polymer solar cells with a solution-processed zinc oxide thin film as an electron collection layer. Sci China Chem, 2012, 5: 755-759

31 O'Brien G A, Quinn A J, Tanner D A, et al. Single polymer nanowire photodetector. Adv Mater, 2006, 18: 2379-2383

32 Wang K, Chen J, Zhou W, et al. Direct growth of highly mismatched type II $\mathrm{ZnO} / \mathrm{ZnSe}$ core/shell nanowire arrays on transparent conducting oxide substrates for solar cell applications. Adv Mater, 2008, 20: 3248-3253

33 Tok Jeffrey B H, Bao Z N. Recent advance I flexible and stretchable electronics, sensors and power sources. Sci China Chem, 2012, 5: 718-725

34 Lauhon L J, Gudiksen M S, Wang D, et al. Epitaxial core-shell and core-multishell nanowire heterostructures. Nature, 2002, 420: 57-61

35 Zhang Y, Ichihashi T, Landree E, et al. Heterostructures of single- walled carbon nanotubes and carbide nanorods. Science, 1999, 285 : 1719-1722

36 Xue F H, Fei G T, Wu B, et al. Direct electrodeposition of highly dense $\mathrm{Bi} / \mathrm{Sb}$ superlattice nanowire arrays. J Am Chem Soc, 2005, 127 : 15348-15349

37 Wang Q, Wang G, Xu B, et al. Non-aqueous cathodic electrodeposition of large-scale uniform $\mathrm{ZnO}$ nanowire arrays embedded in anodic alumina membrane. Mater Lett, 2005, 59: 1378-1382

38 Ostermann R, Li D, Yin Y, et al. $\mathrm{V}_{2} \mathrm{O}_{5}$ nanorods on $\mathrm{TiO}_{2}$ nanofibers: A new class of hierarchical nanostructures enabled by electrospinning and calcination. Nano Lett, 2006, 6: 1297-1302

39 Mieszawska A J, Jalilian R, Sumanasekera G U, et al. The synthesis and fabrication of one-dimensional nanoscale heterojunctions. Small, 2007, 3: 722-756

40 Park S, Lim J H, Chung S W, et al. Self-assembly of mesoscopic metal-polymer amphiphiles. Science, 2004, 303: 348-351

41 Lahav M, Weiss E A, Xu Q, et al. Core-shell and segmented polymer-metal omposite nanostructures. Nano Lett, 2006, 6: 2166-2171

42 Cao H, Tie C, Xu Z, et al. Array of nickel nanowires enveloped in polyaniline nanotubules and its magnetic behavior. Appl Phy Lett, 2001, 78: 1592-1594

43 Guo Y, Tang Q, Liu H, et al. Light-controlled organic/inorganic p-n junction nanowires. J Am Chem Soc, 2008, 130: 9198-9199

44 Chen N, Qian X, Lin H, et al. Synthesis and characterization of axial heterojunction inorganic-organic semiconductor nanowire arrays. Dalton Trans, 2011, 40: 10804-10808

45 Chen N, Qian X, Lin H, et al. Growing uniform copolymer nanowire arrays for high stability and efficient field emission. J Mater Chem, 2012, 22: 11068-11072

46 Lei Y, Liao Q, Fu H, et al. Orange-blue-orange triblock onedimensional heterostructures of organic microrods for white-light emission. J Am Chem Soc, 2010, 132: 1742-1743

47 Liu H, Zhao Q, Li Y, et al. Field emission properties of large-area nanowires of organic charge-transfer complexes. J Am Chem Soc, 2005, 127: 1120-1121

48 Ouyang C B, Liu H B, Qian X M, et al. Field emission and electrical bistable properties of CuTCPQ nanostructures. Dalton Trans, 2011, 40: 3553-3557

49 Cui S, Li Y, Guo Y, et al. Fabrication and field-emission properties of large-area nanostructures of the organic charge-transfer complex Cu-TCNAQ. Adv Mater, 2008, 20: 309-313

50 Liu H, Cui S, Guo Y, et al. Fabrication of large-area hybrid nanowires arrays as novel field emitters. J Mater Chem, 2009, 19: 10311036

51 Liu H, Liu Z, Qian X, et al. Field emission and electrical switching properties of large-area CuTCNQ nanotube arrays. Crystal Growth Design, 2009, 10: 237-243

52 Huang C, Li Y, Song Y, et al. Ordered nanosphere alignment of porphyrin for the improvement of nonlinear optical properties. Adv Mater, 2010, 22: 3532-3536

53 Kempa T J, Tian B, Kim D R, et al. Single and tandem axial p-i-n nanowire photovoltaic devices. Nano Lett, 2008, 8: 3456-3460

Open Access This article is distributed under the terms of the Creative Commons Attribution License which permits any use, distribution, and reproduction in any medium, provided the original author(s) and source are credited. 